\title{
Ultrasonographic Analysis of Optimal Needle Placement for Extensor Indicis
}

\author{
Jin Young Kim, MD ${ }^{1}$, Hyun Seok, MD $^{1}$, Sang-Hyun Kim, $\mathrm{MD}^{1}$, \\ Yoon-Hee Choi, MD $^{2}$, Jun Young Ahn, MD $^{1}$, Seung Yeol Lee, MD $^{1}$ \\ ${ }^{1}$ Department of Physical Medicine and Rehabilitation, Soonchunhyang University Bucheon Hospital, Soonchunhyang University \\ College of Medicine, Bucheon; \\ ${ }^{2}$ Department of Physical Medicine and Rehabilitation, Soonchunhyang University Seoul Hospital, Soonchunhyang University \\ College of Medicine, Seoul, Korea
}

Objective To determine the most optimal needle insertion point of extensor indicis (EI) using ultrasound. Methods A total 80 forearms of 40 healthy volunteers were recruited. We identified midpoint (MP) of EI using ultrasound and set MP as optimal needle insertion point. The location of MP was suggested using distances from landmarks. Distance from MP to medial border of ulna (MP-X) and to lower margin of ulnar head (MP-Y) were measured. Ratios of MP-X to Forearm circumference (X ratio) and MP-Y to forearm length (Y ratio) were calculated. In cross-sectional view, depth of MP (Dmp), defined as middle value of superficial depth (Ds) and deep depth (Dd) was measured and suggested as proper depth of needle insertion.

Results Mean MP-X was $1.37 \pm 0.14 \mathrm{~cm}$ and mean MP-Y was $5.50 \pm 0.46 \mathrm{~cm}$. Mean X ratio was $8.10 \pm 0.53$ and mean Y ratio was 22.15 \pm 0.47 . Mean Dmp was $7.63 \pm 0.96 \mathrm{~mm}$.

Conclusion We suggested that novel optimal needle insertion point of the EI. It is about $7.6 \mathrm{~mm}$ in depth at about $22 \%$ of the forearm length proximal from the lower margin of the ulnar head and about $8.1 \%$ of the forearm circumference radial from medial border of ulna.

Keywords Extensor indicis, Injections, Ultrasonography, Electromyography

\section{INTRODUCTION}

The extensor indicis (EI) muscle arises from the posterior surface of the distal third of the ulna and the adjacent interosseous membrane [1]. It proceeds diagonally, turn- ing into the tendon and inserting into the index finger via the extensor expansion $[2,3]$. The muscle belongs to the deep layer of the posterior compartment of the forearm [4], located under the extensor digitorum and extensor digiti minimi muscles [5]. It is innervated by the posterior

Received March 3, 2020; Revised June 5, 2020; Accepted June 23, 2020; Published online December 31, 2020

Corresponding author: Seung Yeol Lee

Department of Physical Medicine and Rehabilitation, Soonchunhyang University Bucheon Hospital, Soonchunhyang University College of Medicine, 170 Jomaru-ro, Wonmi-gu, Bucheon14584, Korea. Tel: +82-32-621-5057, Fax: +82-32-621-6148, E-mail: shouletz@schmc.ac.kr

ORCID: Jin Young Kim (http://orcid.org/0000-0002-6323-8927); Hyun Seok (http://orcid.org/0000-0001-7266-6045); Sang-Hyun Kim (http://orcid. org/0000-0003-4475-5571); Yoon-Hee Choi (http://orcid.org/0000-0001-6651-7908); Jun Young Ahn (http://orcid.org/0000-0003-4340-2774); Seung Yeol Lee (http://orcid.org/0000-0003-1571-9408).

(c) This is an open-access article distributed under the terms of the Creative Commons Attribution Non-Commercial License (http://creativecommons.org/ licenses/by-nc/4.0) which permits unrestricted noncommercial use, distribution, and reproduction in any medium, provided the original work is properly cited. Copyright (C) 2020 by Korean Academy of Rehabilitation Medicine 
interosseous nerve, which is a branch of the radial nerve, and receives its blood supply from the posterior interosseous artery $[4,6]$.

The EI is used clinically for diagnosis and treatment of several diseases. In needle electromyography (EMG), the $\mathrm{EI}$ is important for diagnosis of radial nerve or $\mathrm{C} 7$ and C8 nerve root lesions. The EI is also used as electrode attachment site for the compound muscle action potentials of the radial nerve in nerve conduction studies. The EI is a target muscle for botulinum toxin injection in patients with hand dystonia or post-stroke spasticity of the upper extremities $[7,8]$. Trigger point injection of the EI is often performed in patients with myofascial syndrome caused predominantly by finger overuse [9].

An optimal needle insertion position for EI has been suggested by several EMG methods [10-12]. However, the needle insertion positions of these methods differ from each other. Because the EI is thin and covered by the superficial layer muscles of the forearm [4], it is difficult to precisely insert a needle into the EI. Several previous studies showed that the accuracy of needle insertion into the EI is low [13,14]. Im et al. [2] attempted to identify the optimal needle insertion point for the EI in cadavers. However, the proper depth of needle placement was not discussed. Also, there was a limitation in that living musculoskeletal structures may differ from those in cadavers. Aside from the cadaveric study, there have not been any studies on optimal needle placement in the EI in living human bodies.

The purpose of this study is to determine the optimal needle insertion point considering the anatomical location of the EI with surrounding structures using ultrasound.

\section{MATERIALS AND METHODS}

\section{Study design}

This was a cross-sectional study that investigated the proper needle insertion point in the EI using ultrasound.

\section{Subjects}

Forty healthy volunteers were recruited for this study. Both forearms were measured in all subjects, so a total of 80 forearms were examined. Upper extremity amputation patients and subjects who had a cast, splint, or artifactproducing metal fixture in the forearm were excluded.
Persons with previous surgery on the forearm were also excluded, because the operation might cause structural changes in the forearm muscles. Demographic characteristics, including age, sex, height, weight, and body mass index (BMI), were collected.

All participants received a comprehensive explanation of the study and signed an informed consent form. The present study was approved by the Institutional Review Board of Soonchunhyang University Buchoen Hospital (No. 2019-10-002-001).

\section{Ultrasonographic examination}

All subjects underwent ultrasonographic evaluation in the supine position with the forearm fully pronated, the elbow flexed about $30^{\circ}$, and the shoulder abducted about $30^{\circ}$. Before ultrasonographic evaluation, wrist circumference and forearm length were measured in this position. Forearm length was defined as the vertical distance from the elbow crease to the lower margin of the ulnar head. Wrist circumference was measured at the level of the lower margin of the ulnar head.

After measurements were taken, ultrasonographic evaluation was performed by a physiatrist with more than 5 years of experience with musculoskeletal ultrasound, using a XARIO (SSA-660A; Toshiba, Minato, Japan) with a 7-18 MHz linear array transducer (Toshiba). Because the EI shows a fusiform-shaped muscle belly [15], the midpoint of the EI (MP), which can easily be identified and targeted, was suggested as an optimal needle insertion point in this study. The vertical location of MP was defined as the point midway between the proximal origin (PO) and the musculotendinous junction (MTJ) of the EI. To determine the location of MP, the PO and MTJ of the EI were first identified on short-axis view by moving a probe proximally from the lower margin of the ulnar head. The locations of the PO and MTJ on shortaxis view were confirmed on long-axis view. The PO and MTJ were marked on the skin, as shown in Fig. 1A. MP was investigated by moving the probe horizontally midway between the PO and MTJ. The point where the EI was located on the midline on short-axis view was set as MP. MP was also marked on the skin and forearm circumference at the level of MP was measured. To represent MP location, the medial border of the ulna was set as a landmark of horizontal distance and the lower margin of the ulnar head was used as a landmark of vertical distance. 

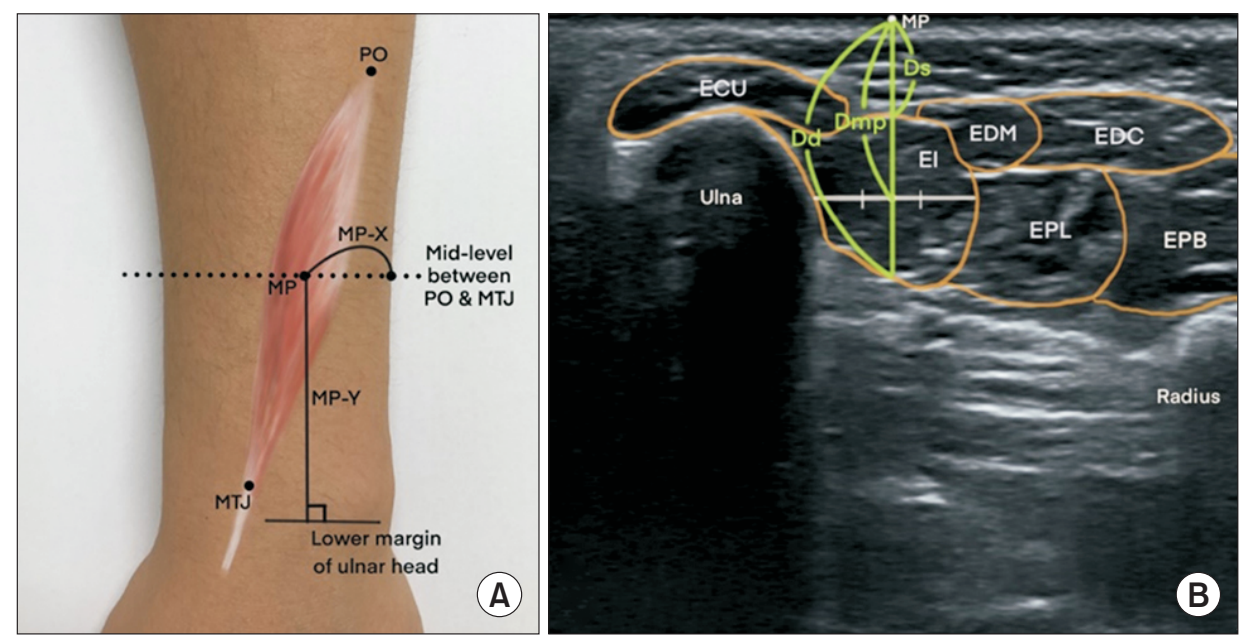

Fig. 1. Parameters measured for midpoint of extensor indicis. (A) Schematic diagram of extensor indicis in forearm. (B) Short-axis ultrasound image of midpoint. MP, midpoint; PO, proximal origin; MTJ, musculotendinous junction; MPY, distance from midpoint to lower margin of ulnar head; MP-X, distance from midpoint to medial border of ulna; Ds, duperficial depth; Dd, deep depth; Dmp, midpoint depth; EI, extensor indicis; EDM, extensor digiti minimi; EDC, extensor digitorum communis; EPL, extensor pollicis longus; EPB, extensor pollicis brevis; ECU, extensor carpi ulnaris.
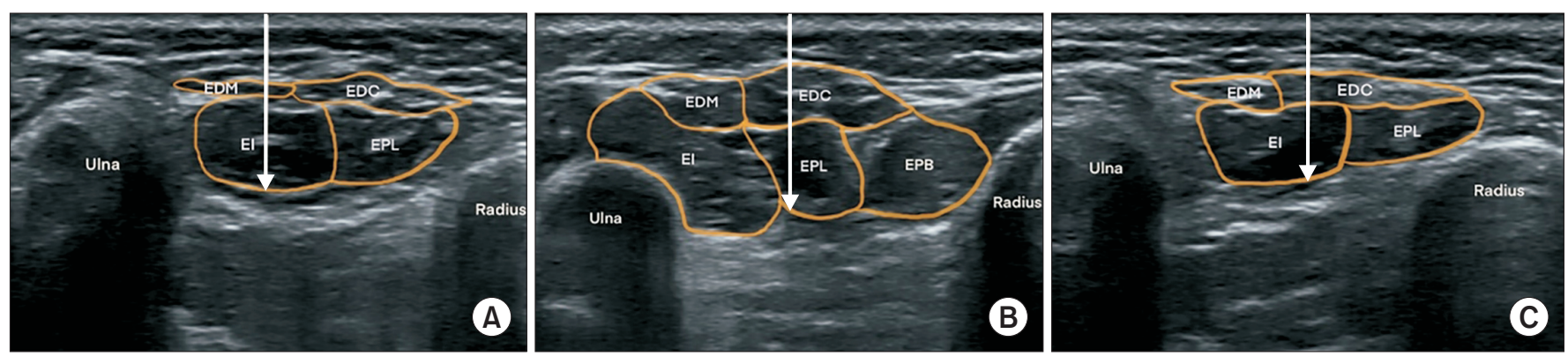

Fig. 2. Cross-sectional ultrasound images of three EMG methods: (A) point A, (B) point B, and (C) point C. White arrow indicates needle pathway. EI, extensor indicis; EDM, extensor digiti minimi; EDC, extensor digitorum communis; EPL, extensor pollicis longus; EPB, extensor pollicis brevis.

The distances from MP to the medial border of the ulna (MP-X) and to the lower margin of the ulnar head (MP-Y) were measured (Fig. 1A). In order to indicate MP position relative to forearm circumference and length, the ratios of MP-X to forearm circumference (X ratio) and MP-Y to forearm length ( $\mathrm{Y}$ ratio) were calculated as percentages. Using these measurements, the distances and ratios of MP were obtained. On a cross-sectional view of MP (Fig. 1B), the superficial depth (Ds) and deep depth (Dd) of the EI were measured. The depth of MP (Dmp) was defined as the median value of the Ds and Dd and suggested as the optimal depth for needle insertion.

There are several existing needle insertion methods based on EMG. Perotto et al. [10] suggested needle inser- tion at two finger breadths proximal to the ulnar styloid process, just radial to the ulna at a depth of one-half inch (Point A). Lee and DeLisa [11] recommended inserting the needle in the distal fourth of the forearm lateral to the radial side of the ulna between the extensor digitorum and extensor carpi ulnaris tendons (Point B). ChuAndrews and Johnson [12] proposed needle insertion at $2.5 \mathrm{~cm}$ proximal to the lower border of the styloid process of the ulna, in line with the lateral aspect of the ulna with the forearm pronated (Point $\mathrm{C}$ ). Needle location and muscle penetration using these methods were analyzed in this study. With the three existing needle EMG methods, each needle insertion point is marked on the skin. On cross-sectional view at each point (Fig. 2), using an 
imaginary needle pathway, whether the needle can be inserted in the EI, the needle location in the EI if insertion is possible, and the other muscles that could be involved were investigated. Needle location in the EI was classified into the middle portion or the other portion. The middle portion of the EI is defined as the middle third when the muscle is divided into three parts. Needle insertion in the EI or other muscles were recorded. The distances from each insertion point to MP in the three different methods were also measured.

In order to accurately distinguish between different muscles, ultrasound was performed while contraction of

Table 1. Demographic data of participants $(n=40)$

\begin{tabular}{lc}
\hline \multicolumn{1}{c}{ Characteristic } & Value \\
\hline Age $(\mathrm{yr})$ & $30.7 \pm 6.8(24-51)$ \\
Sex & 20 \\
$\quad$ Male & 20 \\
Female & $168.9 \pm 10.8(151.0-189.0)$ \\
Height $(\mathrm{cm})$ & $64.7 \pm 12.7(43.0-88.0)$ \\
Weight $(\mathrm{kg})$ & $22.5 \pm 2.7(17.5-29.3)$ \\
Body mass index $\left(\mathrm{kg} / \mathrm{m}^{2}\right)$ & $24.8 \pm 2.1(21.3-29.4)$ \\
Forearm length $(\mathrm{cm})$ & $15.4 \pm 1.3(13.8-20.9$ \\
Wrist circumference $(\mathrm{cm})$ & \\
Examined forearm & 40 \\
$\quad$ Right & 40 \\
\hline Left & \\
\hline
\end{tabular}

Values are presented as mean \pm standard deviation (range). surrounding muscles, such as the EI, extensor digitorum, extensor digiti minimi, and extensor pollicis longus was induced. During ultrasound examination, the probe was held perpendicular to the muscle with minimal pressure to ensure accurate measurement.

\section{Statistical analysis}

Anatomical and ultrasound parameters and demographic data are represented as mean \pm standard deviation. Normality of data distribution was confirmed using the Shapiro-Wilk test. ANOVA and Student t-test were used to compare parameters between groups. Bonferroni post-hoc test was performed to identify the differences in MP-X and MP-Y between the groups. To compare the accuracy of each EMG method between height groups, the chi-square test was conducted. A p-value of 0.05 or less was determined to be statistically significant. All analyses were executed using SPSS software ver. 19.0 (IBM, Armonk, NY, USA).

\section{RESULTS}

A total of 80 forearms of 40 participants (20 men and 20 women) were examined. The mean age was $30.7 \pm 6.8$ years and the mean height was $168.9 \pm 10.8 \mathrm{~cm}$. Detailed demographic data are presented in Table 1.

Anatomical and sonographic measurements are summarized in Table 2. Mean forearm length was 24.83 \pm 2.12 $\mathrm{cm}$ and forearm circumference at MP was $16.96 \pm 1.67 \mathrm{~cm}$.

Table 2. Anatomical and sonographic measurements

\begin{tabular}{|c|c|c|c|c|c|c|c|}
\hline & Total $(n=80)$ & Right $(n=40)$ & Left $(n=40)$ & p-value ${ }^{\text {a) }}$ & Men $(n=40)$ & Women $(n=40)$ & p-value ${ }^{a)}$ \\
\hline $\mathrm{FL}(\mathrm{cm})$ & $24.83 \pm 2.12$ & $24.82 \pm 2.16$ & $24.83 \pm 2.11$ & 0.983 & $26.53 \pm 1.31$ & $23.12 \pm 1.21$ & $<0.0001$ \\
\hline $\mathrm{FC}(\mathrm{cm})$ & $16.96 \pm 1.67$ & $16.97 \pm 1.68$ & $16.95 \pm 1.68$ & 0.958 & $17.94 \pm 1.25$ & $15.99 \pm 1.46$ & $<0.0001$ \\
\hline MP-Y (cm) & $5.50 \pm 0.46$ & $5.51 \pm 0.48$ & $5.49 \pm 0.45$ & 0.886 & $5.86 \pm 0.32$ & $5.14 \pm 0.24$ & $<0.0001$ \\
\hline Y ratio (\%) & $22.15 \pm 0.47$ & $22.18 \pm 0.45$ & $22.12 \pm 0.50$ & 0.564 & $22.09 \pm 0.52$ & $22.22 \pm 0.41$ & 0.231 \\
\hline MP-X (cm) & $1.37 \pm 0.14$ & $1.37 \pm 0.15$ & $1.37 \pm 0.12$ & 0.935 & $1.45 \pm 0.12$ & $1.29 \pm 0.10$ & $<0.0001$ \\
\hline X ratio (\%) & $8.10 \pm 0.53$ & $8.10 \pm 0.60$ & $8.10 \pm 0.46$ & 0.988 & $8.11 \pm 0.56$ & $8.09 \pm 0.50$ & 0.873 \\
\hline Ds (mm) & $4.40 \pm 0.97$ & $4.38 \pm 0.93$ & $4.42 \pm 1.02$ & 0.864 & $4.04 \pm 1.00$ & $4.76 \pm 0.81$ & 0.001 \\
\hline $\mathrm{Dd}(\mathrm{mm})$ & $10.85 \pm 1.31$ & $10.80 \pm 1.30$ & $10.91 \pm 1.34$ & 0.723 & $11.2 \pm 1.39$ & $10.5 \pm 1.16$ & 0.024 \\
\hline $\operatorname{Dmp}(\mathrm{mm})$ & $7.63 \pm 0.96$ & $7.59 \pm 0.93$ & $7.66 \pm 0.99$ & 0.741 & $7.61 \pm 1.00$ & $7.64 \pm 0.91$ & 0.903 \\
\hline
\end{tabular}

Values are presented as mean \pm standard deviation.

FL, forearm length; FC, forearm circumference; MP-Y, distance from midpoint to lower margin of ulnar head; Y-ratio, MP-Y/forearm length; MP-X, distance from midpoint to medial border of ulna; X-ratio, MP-X/forearm circumference; Ds, superficial depth; Dd, deep depth; Dmp, midpoint depth.

${ }^{a)}$ By Student t-test. 
The MP-Y was $5.50 \pm 0.46 \mathrm{~cm}$ and the mean $\mathrm{Y}$ ratio was $22.15 \pm 0.47$. The MP-X was $1.37 \pm 0.14 \mathrm{~cm}$ and the mean $\mathrm{X}$ ratio was $8.10 \pm 0.53$. The Ds and Dd of the EI at MP were $4.40 \pm 0.97 \mathrm{~mm}$ and $10.85 \pm 1.31 \mathrm{~mm}$, respectively, and Dmp was $7.63 \pm 0.96 \mathrm{~mm}$. There were significant differences in forearm length, forearm circumference, MP-Y, MP-X, Ds, and Dd between men and women. However, the $\mathrm{Y}$ ratio, $\mathrm{X}$ ratio, and Dmp did not significantly differ between the sexes. There were no significant differences between the right and left sides in any measurements.

The participants were categorized into four groups according to height. MP-Y, Y ratio, MP-X, and X ratio of each group are shown in Table 3. MP-Y and MP-X showed a tendency to increase as height increased, whereas the $\mathrm{Y}$ ratio and $\mathrm{X}$ ratio did not. The $\mathrm{Y}$ ratio and $\mathrm{X}$ ratio did not show statistically significant differences between groups. However, MP-Y and MP-X significantly differed between groups.

Post-hoc tests revealed no significant differences in MP-X between the 150s and 160s groups ( $\mathrm{p}=0.105), 160 \mathrm{~s}$ and 170 s groups $(\mathrm{p}=0.063)$, and $170 \mathrm{~s}$ and 180 s groups $(p=0.558)$. On the other hand, there were significant differences in MP-X between the 150s and 170s groups $(\mathrm{p}<0.0001), 150$ s and 180 s groups $(\mathrm{p}<0.0001)$, and $160 \mathrm{~s}$ and 180s groups ( $\mathrm{p}=0.001)$. In post-hoc analysis of MP-Y, significant differences were identified between all groups ( 150 vs. $160, p=0.007$; others, $\mathrm{p}<0.0001$ ).

The structures into which the needle could be inserted according to the three EMG methods are shown in Table 4. Short-axis ultrasound images of points on the skin taken using the three methods are shown in Fig. 2. The probability that the needle course passes the EI was $100 \%, 50 \%$, and $100 \%$ and the probability that the needle crosses the middle portion of the EI was $52.5 \%, 16.25 \%$, and $42.5 \%$ for points A, B, and C, respectively. In these three points, the needle had to penetrate other muscles in $100 \%$ of cases in order for the needle to reach the EI. The most often penetrated muscle was the extensor digiti minimi in all three methods. The mean distances between MP and each point on the skin were $2.43 \pm 0.65$ $\mathrm{cm}, 1.20 \pm 0.37 \mathrm{~cm}$ and $3.34 \pm 0.71 \mathrm{~cm}$. Point B was closest and point $C$ was farthest away (Table 4). For each method, the differences in accuracy of needle placement between height groups were analyzed. Needle placement was divided depending on whether the needle passed through the middle portion of the EI, other portion of the

Table 3. Vertical and horizontal distance of midpoint (MP-Y and MP-X) and ratio to forearm (Y ratio and X ratio) according to height groups

\begin{tabular}{|c|c|c|c|c|c|}
\hline & \multicolumn{4}{|c|}{ Height $(\mathrm{cm})$} & \multirow{2}{*}{ p-value ${ }^{a)}$} \\
\hline & $150-159(n=22)$ & $160-169(n=20)$ & $170-179(n=22)$ & $180-189(n=16)$ & \\
\hline MP-Y (cm) & $5.05 \pm 0.19$ & $5.25 \pm 0.24$ & $5.67 \pm 0.17$ & $6.18 \pm 0.16$ & $<0.0001$ \\
\hline Y ratio (\%) & $22.22 \pm 0.40$ & $22.19 \pm 0.50$ & $21.99 \pm 0.54$ & $22.24 \pm 0.42$ & 0.513 \\
\hline $\mathrm{MP}-\mathrm{X}(\mathrm{cm})$ & $1.26 \pm 0.08$ & $1.34 \pm 0.11$ & $1.43 \pm 0.12$ & $1.49 \pm 0.11$ & $<0.0001$ \\
\hline $\mathrm{X}$ ratio $(\%)$ & $8.08 \pm 0.41$ & $8.09 \pm 0.68$ & $8.12 \pm 0.59$ & $8.14 \pm 0.39$ & 0.987 \\
\hline
\end{tabular}

Values are presented as mean \pm standard deviation.

MP-Y, distance from midpoint to lower margin of ulnar head; Y-ratio, MP-Y/forearm length; MP-X, distance from midpoint to medial border of ulna; X-ratio, MP-X/forearm circumference.

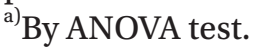

Table 4. Structures that could be inserted in different three EMG methods based on short-axis ultrasound image and distance from midpoint to each needle insertion points

\begin{tabular}{ccccccc}
\hline Point & EI (middle+others) & EI (middle) & EDM & EDC & EPL & Distance from MP (cm) \\
\hline A & $80(100)$ & $42(52.5)$ & $59(73.75)$ & $21(26.25)$ & $0(0)$ & $2.43 \pm 0.65$ \\
B & $40(50)$ & $13(16.25)$ & $49(61.25)$ & $31(38.75)$ & $40(50)$ & $1.20 \pm 0.37$ \\
C & $80(100)$ & $34(42.5)$ & $45(56.25)$ & $35(43.75)$ & $0(0)$ & $3.34 \pm 0.71$ \\
\hline
\end{tabular}

Values are presented as number of inserted (\% of the number of inserted over total number).

EMG, electromyography; MP, midpoint; EI, extensor indicis; EDM, extensor digiti minimi; EDC, extensor digitorum communis; EPL, extensor pollicis longus. 
EI, or not at all. In all methods, there were no significant between-group differences (point $\mathrm{A}, \mathrm{p}=0.809$; point $\mathrm{B}$, $\mathrm{p}=0.109$; point $\mathrm{C}, \mathrm{p}=0.450$ ).

\section{DISCUSSION}

In this study, a new needle insertion method for the EI using ultrasound is proposed. We demonstrated that the MP of the EI was located on average about $5.5 \mathrm{~cm}$ proximal from the lower margin of the ulnar head and about $1.4 \mathrm{~cm}$ radial from the medial border of the ulna. We also identified that if a needle is inserted about $7.6 \mathrm{~mm}$ deep at about $22 \%$ of the forearm length from the lower margin of the ulnar head and about $8.1 \%$ of the forearm circumference from the medial border of the ulna, it will be placed in MP of the EI. Studies have been done on the optimal location of needle insertion in various muscles based on ultrasound, but to the best of our knowledge, this is the first study to investigate needle insertion position in the EI using ultrasound.

The EI is used for diagnosis of several neurologic lesions in needle EMG and treatment of spasticity or myofascial syndrome using botulinum toxin injection or trigger point injection. If the needle is placed incorrectly, a misdiagnosis can be made or the therapeutic effect may be diminished. For example, unintended muscle weakness can occur after botulinum toxin injection due to inaccurate needle insertion. Nevertheless, needle EMG or injection is usually performed blindly in practice because using ultrasound or computed tomography to ensure needle placement requires a large investment in time and cost. Therefore, it is very important to establish a method for precise needle insertion into the EI that is applicable to patients in general.

Accurate needle insertion in the EI is challenging. There are several reasons why it is so difficult. First, the EI is thin and runs diagonally [2]. Thus, even a slight change in needle position may result in incorrect needle insertion. As the EI runs distally, it becomes a tendon and the thickness of the belly decreases, which makes it difficult to place the needle in this muscle when insertion is in the distal part. If the needle is inserted too radially or proximally, it may end up in the abductor pollicis longus or extensor pollicis longus $[10,11]$. Needle insertion distal to the EI may lead to positioning in the tendon of the EI [2]. Second, the EI is covered by superficial muscles of the forearm and is not prominent because of its small size. Muscles in the posterior compartment of the forearm can be divided into a superficial layer and a deep layer. The superficial layer includes the brachioradialis, extensor carpi radialis longus, extensor carpi radialis brevis, extensor carpi ulnaris, anconeus, extensor digitorum, and extensor digiti minimi, whereas the deep layer includes the extensor indicis, abductor pollicis longus, extensor pollicis longus, extensor pollicis brevis, and supinator [4]. The EI is positioned under superficial layer muscles such as the extensor digiti minimi and extensor digitorum communis and does not protrude anatomically, unlike the thenar or hypothenar muscles. Therefore, it is difficult to insert a needle after confirming the contour of the muscle through palpation. Previous studies on the accuracy of blind needle insertion for muscles in the extremities have shown that deep muscles are associated with lower insertion accuracy compared to superficial muscles, and smaller muscles have lower insertion accuracy than larger muscles [13]. The needle can be placed in the extensor digiti minimi or extensor carpi ulnaris after superficial insertion or the pronator quadratus after deep insertion $[13,16]$. Third, because the EI is not clearly separated from other muscles that are innervated by the radial nerve, it is difficult to distinguish the EI from other muscles [17]. Due to the characteristics of the EI, a muscle contraction is often induced to identify the location of the muscle and insert the needle exactly. However, a large number of patients with radial neuropathy, who commonly undergo needle EMG, show decreased muscle strength in the index finger. So, it is difficult to palpate the EI using muscle contraction in these patients. Since patients with post-stroke spasticity have difficulty with selective finger movement, co-contraction of the forearm muscles hinders the effort to identify the location of the EI [18].

Previous studies showed that correct needle insertion in the EI is not easy. Karvelas et al. [14] investigated the accuracy of needle EMG of the EI, pronator teres, peroneus longus, and soleus when third- and fourth-year medical residents performed needle EMG in live subjects. The accuracy of insertion into the EI was $20 \%-42 \%$, which was the lowest value among the examined muscles. In a cadaveric study, Goodmurphy et al. [13] proved that the EI, serratus anterior, flexor carpi ulnaris, and flexor carpi radialis were the most difficult to properly insert a needle 
into among the muscles of the upper extremities.

Several needle EMG methods for the EI have been proposed. The probability that the needle pathway passes through the EI was $100 \%$ at points A and C. However, the probability of passing the middle portion was not high, at just $52.5 \%$ and $42.5 \%$, respectively. Because simply piercing the muscle does not imply correct needle placement in the EI and the belly is thinner in the distal portion, the accuracy of needle insertion methods performed at the distal portion, such as points $\mathrm{A}$ and $\mathrm{C}$, may be lower than $100 \%$. Finger breadth is used as a measurement tool in point $\mathrm{A}$, but this is not an objective tool because it differs between examiners. Point $C$ uses an objective value of 2.5 $\mathrm{cm}$, which is difficult to apply to everyone because each person has a different forearm length. Point $B$ was closest to the MP, but showed the lowest probability of the needle pathway crossing the EI or crossing the middle portion of the EI among the three methods (50\% and $16.25 \%$, respectively). In the remaining $50 \%$ of cases, in which the needle did not pass the EI, the needle pathway pierced the extensor pollicis longus, which is located directly radial to the EI. Therefore, the low accuracy at point B may be because the needle is not inserted into the EI even if the transverse position is slightly altered due to the elongated shape of the EI.

The mean distance from MP to each of the skin points is presented in this study (Table 4). These points were located in different directions from MP. Points A and C were distal and radial from MP. The needle pathways at points A and C penetrated the EI in all cases. Therefore, the depth is more important than the horizontal and vertical distances when inserting the needle at points $\mathrm{A}$ and C. Unlike points A and C, point B was located proximally and radially from MP. The needle pathway at point $B$ passed through the EI in only $50 \%$ of cases. In the $50 \%$ of cases in which the needle did not pass through the EI, the needle pathway penetrated the extensor pollicis longus, which is located on the radial side of the EI. If the needle is not positioned correctly in the $\mathrm{EI}$ at point $\mathrm{B}$, the needle should be repositioned toward the ulnar side.

MP presented in this study has several advantages over previous methods. At points A, B, and C, the needle could reach the EI only by penetrating the superficial layer muscles, extensor digiti minimi, or extensor digitorum communis in all examined forearms (Fig. 2). On the other hand, in the method proposed in this study, the EI is su- perficial in all subjects as confirmed by a cross-sectional view (Fig. 1B). If the needle is inserted at a superficial point, it is possible to better palpate the muscle and place the needle more precisely. Also, less pain occurs when the muscle is contracted with the needle in place. Points $\mathrm{A}, \mathrm{B}$, and $\mathrm{C}$ did not provide accurate horizontal distances, but in our method, the horizontal position is presented as a ratio of forearm circumference and is applicable regardless of height. Safwat and Abdel-Meguid [19] demonstrated that the motor point of the EI is in the middle third in $100 \%$ of cases. Thus, there is a high possibility of needle placement at the motor point during insertion in MP. However, the needle is inserted at the distal EI at points $\mathrm{A}$ and $\mathrm{C}$, making it difficult to position the needle at the motor point. Given these advantages, it is better to insert needles in MP than by using conventional methods.

The current study demonstrated that the horizontal distance of the optimal insertion point was about $8.1 \%$ of the forearm circumference in all height groups. However, it is difficult and time consuming to calculate the percentage of forearm circumference in a clinical setting. Post-hoc test showed that there was no significant difference in MP-X between the 150s and 160s groups, 160s and 170s groups, and 170s and 180s groups. The difference in average of MP-X (about $0.9 \mathrm{~cm}$ ) between the $160 \mathrm{~s}$ and 170s groups was larger than the differences between the other groups, and the corresponding p-value was also the largest. Thus, it may be a good alternative to apply the average MP-X above and below a height cutoff value of $170 \mathrm{~cm}$. Thus, $1.3 \mathrm{~cm}$ radial from the medial border of the ulna can be applied to the under- $170 \mathrm{~cm}$ group and 1.5 $\mathrm{cm}$ can be used in the over-170 $\mathrm{cm}$ group as the horizontal distance of MP.

In this study, the average MP-Y and Y ratio of all participants were about $5.5 \mathrm{~cm}$ and $22 \%$. MP-Y shows significant differences between height groups. Unlike MP-X, there were also significant differences in MP-Y between all adjacent groups. Therefore, it is difficult to suggest MP-Y as absolute value, not as percentage. However the absolute value is easier to apply in the clinical practice than a percentage of forearm length so further studies about the novel method that can propose the horizontal distance as absolute value are needed.

Im et al. [2] proposed that the midpoint of the EI is an appropriate needle insertion point in a cadaveric study. 
They suggested the position of the midpoint at about 4.8 $\mathrm{cm}$ (about $21.1 \%$ of the forearm length) proximal from the ulnar styloid process and about $0.7 \mathrm{~cm}$ (about $12.6 \%$ of the forearm width at the midpoint) from the medial border of the ulna. By our calculations, the MP-Y was about $5.5 \mathrm{~cm}$, which was different from theirs, but the ratio of MP-Y to forearm length was $22.0 \%$, which was similar. The difference between these studies might be attributable to a change in the length and thickness of the muscles during the process of embalming the cadavers.

In addition, the previous study did not include cadavers with various heights, so a selection bias may have occurred. In the cadaveric study, the horizontal distance of the midpoint was presented as a straight line rather than a circumference, which makes it difficult to compare directly with the MP-X. In actual clinical trials, it is difficult to use the linear distance during needle insertion and it is more useful to use the circumference. The depth is important for accurate needle insertion into the muscles of the forearm, which has many small muscles with multiple layers, but the previous study did not investigate depth. The styloid process of the ulna was used as the landmark for vertical distance in the cadaveric study. However, the location of the ulnar styloid process can be confusing because the ulnar styloid process can be located on either the volar or dorsal side when the forearm is pronated or supinated [20] and may not be palpable in some people. The ulnar head, on the other hand, is easily palpable in most people, even overweight people, regardless of posture and is the most prominent structure in the wrist, making it a better landmark.

There were several limitations of this study. The subjects did not include people with other diseases such as neurologic lesions or strokes. Muscle atrophy due to denervation can occur in patients with neurologic lesions and disuse atrophy can occur in stroke patients. If such morphologic changes occur, accurate needle insertion may be difficult with the method presented in this study. Future studies are needed to target patients with various diseases and conduct subgroup analysis. Second, variations in the EI have been reported in some studies. According to several previous studies, there are extensor medii proprius or digiti, extensor indicis et medii communis or digiti, extensor pollicis et indicis, and extensor indicis ulnaris and radialis muscles $[21,22]$. In people with these variations, it may be difficult to identify the optimal insertion point using our method. In the present study, there were no participants with variations of the EI. Third, in the present study, the total mean BMI was $22.5 \pm 2.7 \mathrm{~kg} / \mathrm{m}^{2}$ and the range was $17.5-29.3 \mathrm{~kg} / \mathrm{m}^{2}$. Although participants with a variety of BMIs enrolled in the study, the depth of needle insertion may vary in extremely overweight or underweight people. Future studies should include a more varied population. Lastly, the nerves and blood vessels adjacent to the EI were not considered in the current study. The posterior interosseous artery and posterior interosseous nerve pass around the EI. Therefore, a safer needle insertion method could be suggested by analyzing the locational relationship between MP and the surrounding vessels or nerves.

In conclusion, various needle insertion methods for the EI have been proposed, but each has several limitations. In this study, we propose a new and accurate needle insertion method for the EI using ultrasound. The optimal needle placement point for EI, which avoids penetrating other muscles, lies at approximately $22 \%$ of the forearm length from the lower margin of the ulnar head and approximately $8.1 \%$ of the forearm circumference from the medial border of the ulna at a depth of $7.6 \mathrm{~mm}$. We hope that this method will provide more accurate needle EMG or injection treatment for the EI.

\section{CONFLICT OF INTEREST}

No potential conflict of interest relevant to this article was reported.

\section{ACKNOWLEDGMENTS}

This work was supported by the Soonchunhyang University Research Fund.

\section{AUTHOR CONTRIBUTION}

Conceptualization: Kim JY, Lee SY. Methodology: Kim JY, Choi YH, Lee SY. Data collection: Kim JY, Ahn JY. Formal analysis: Kim JY, Lee SY. Project administration: Kim JY, Seok H, Kim SH, Lee SY. Writing - original draft: Kim JY, Writing - review and editing: Kim JY, Seok H, Kim SH, Lee SY. Approval of final manuscript: all authors. 


\section{REFERENCES}

1. Netter FH. Atlas of human anatomy, 6th ed. Philadelphia, PA: Saunders/Elsevier; 2014.

2. Im JY, Park HB, Lee SJ, Lim SG, Kim KH, Kim D, et al. Optimal placement of needle electromyography in extensor indicis: a cadaveric study. Ann Rehabil Med 2018;42:473-6.

3. Komiyama M, Nwe TM, Toyota N, Shimada Y. Variations of the extensor indicis muscle and tendon. J Hand Surg Br 1999;24:575-8.

4. Standring S. Gray's anatomy: the anatomical basis of clinical practice. 41 st ed. St. Louis, MO: Elsevier Health Sciences; 2015.

5. Neumann DA. Kinesiology of the musculoskeletal system: foundations for rehabilitation. 2nd ed. St. Louis, MO: Elsevier Health Sciences; 2013.

6. Leis AA, Trapani VC. Atlas of electromyography. New York, NY: Oxford University Press; 2000.

7. Pandey S. A practical approach to management of focal hand dystonia. Ann Indian Acad Neurol 2015;18:146-53.

8. Thomas AM, Simpson DM. Contralateral weakness following botulinum toxin for poststroke spasticity. Muscle Nerve 2012;46:443-8.

9. Dommerholt J, Fernandez de las Penas C. Trigger point dry needling: an evidence and clinical-based approach. 2nd ed. St. Louis, MO: Elsevier Health Sciences; 2018.

10. Perotto AO, Delagi EF, Hammond PB, Thomas H. Anatomical guide for the electromyographer: the limbs and trunk. 5th ed. Springfield, IL: Charles C. Thomas Publisher; 2011.

11. Lee HJ, DeLisa JA. Manual of nerve conduction study and surface anatomy for needle electromyography. 4th ed. Philadelphia, PA: Lippincott Williams \& Wilkins; 2005.

12. Chu-Andrews J, Johnson RJ. Electrodiagnosis: an anatomical and clinical approach. Philadelphia, PA: Lip- pincott; 1986.

13. Goodmurphy C, Chiodo A, Haig A. The accuracy of needle placement in extremity muscles: a blinded study. J Clin Neurophysiol 2007;24:366-78.

14. Karvelas K, Ziegler C, Rho ME. Resident accuracy of electromyography needle electrode placement using ultrasound verification. PM R 2016;8:748-53.

15. Tweedie A, Rigby E, Cruveilhier J. The library of Medicine. London: Whittaker; 1841.

16. Preston DC, Shapiro BE. Electromyography and neuromuscular disorders: clinical-electrophysiologic correlations. St. Louis, MO: Elsevier Health Sciences; 2012.

17. Yeo J, Kim Y, Kim S, Oh K, Kang H. Optimal radial motor nerve conduction study using ultrasound in healthy adults. Ann Rehabil Med 2017;41:290-8.

18. Molloy FM, Shill HA, Kaelin-Lang A, Karp BI. Accuracy of muscle localization without EMG: implications for treatment of limb dystonia. Neurology 2002;58:805-7.

19. Safwat MD, Abdel-Meguid EM. Distribution of terminal nerve entry points to the flexor and extensor groups of forearm muscles: an anatomical study. Folia Morphol (Warsz) 2007;66:83-93.

20. Shin SH, Lee YS, Kang JW, Noh DY, Jung JY, Chung YG. Where is the ulnar styloid process?: identification of the absolute location of the ulnar styloid process based on CT and verification of neutral forearm rotation on lateral radiographs of the wrist. Clin Orthop Surg 2018;10:80-8.

21. Yammine K. The prevalence of the extensor indicis tendon and its variants: a systematic review and meta-analysis. Surg Radiol Anat 2015;37:247-54.

22. Georgiev GP, Tubbs RS, Iliev A, Kotov G, Landzhov B. Extensor indicis proprius muscle and its variants together with the extensor digitorum brevis manus muscle: a common classification: clinical significance in hand and reconstructive surgery. Surg Radiol Anat 2018;40:271-80. 